\title{
Epigenetic clocks for gestational age: statistical and study design considerations
}

\author{
Andrew J. Simpkin, Matthew Suderman and Laura D. Howe
}

\begin{abstract}
In this letter to the editor, we highlight some concerns with a recently published method to estimate gestational age at delivery from DNA methylation data. We conduct novel analyses to highlight the implications of different choices in study design and statistical methods for the prediction of phenotypes from methylation data.
\end{abstract}

Keywords: Prediction, DNA methylation, Gestational age at delivery, Epigenetic clock

\section{Letter to the editor}

In two recent articles, Bohlin et al. [1] and Knight et al. [2] develop DNA methylation clocks for gestational age at delivery (GA) using cord and newborn blood. These follow from the clock of Horvath [3], which has been used to obtain a measure of age acceleration, the discrepancy between estimated and chronological age, calculated as the residual when regressing one on the other. Associations between age acceleration and a wide range of phenotypes have been observed, including all-cause mortality [4]. The newly developed GA clocks offer the similar possibility to assess determinants of GA acceleration (GAA) and associations with subsequent outcomes. A recent article in this journal used the GA clock developed by Knight et al. to examine associations with maternal and offspring characteristics [5].

We applied both GA clocks to cord blood DNA methylation data from 863 members of the ARIES cohort [6] in order to test associations of GAA with birth weight. We observed a strong positive correlation with the Bohlin GAA estimate $\left(R=0.20, p=5.3 \times 10^{-9}\right)$ and a comparatively weak correlation with the Knight estimate $(R=0.055, p=0.11)$.

To investigate this discrepancy, we asked how well both clocks estimated GA in ARIES. Although both were correlated with GA (Bohlin $R=0.65$; Knight $R=0.37$ ), these correlations are below those reported in their original publications (Bohlin $R=0.81$; Knight $R=0.91$ ).

\footnotetext{
* Correspondence: laura.howe@bristol.ac.uk
MRC Integrative Epidemiology Unit at the University of Bristol, Population

* Correspondence: laura.howe@bristol.ac.uk
MRC Integrative Epidemiology Unit at the University of Bristol, Population Health Sciences, Bristol Medical School, University of Bristol, Oakfield House, Oakfield Grove, Bristol BS8 2BN, UK
}

(c) The Author(s). 2017 Open Access This article is distributed under the terms of the Creative Commons Attribution 4.0 International License (http://creativecommons.org/licenses/by/4.0/), which permits unrestricted use, distribution, and reproduction in any medium, provided you give appropriate credit to the original author(s) and the source, provide a link to the Creative Commons license, and indicate if changes were made. The Creative Commons Public Domain Dedication waiver (http://creativecommons.org/publicdomain/zero/1.0/) applies to the data made available in this article, unless otherwise stated.

In the case of Bohlin, this is expected given that their training and testing sets were drawn from the same cohort study. This was not the case for Knight. The scatterplot of the Knight test results (Figure 1b of [2]) suggested that the strong correlation may have been driven by the inclusion of data for 183 preterm infants in the test set. We tested this hypothesis by adding these preterm infants to ARIES, increasing the percentage of preterm infants (GA < 37 weeks) from 2.8 to $19.8 \%$. As expected, the correlation of the Knight GA estimate rose from $R=0.37$ in ARIES alone to $R=0.89$.

Given the much lower correlation of the Knight compared to the Bohlin estimate in ARIES, we asked if the difference could be due to differences between their training datasets: GA measured using ultrasound (Bohlin) versus last menstrual period (Knight), mean GA (Bohlin 39.9; Knight 36.9), training samples (1068 for Bohlin; 207 for Knight), 450K CpG sites (Bohlin) versus $27 \mathrm{~K} \mathrm{CpG}$ sites (Knight). Most of these predict that Bohlin will perform best in an average population like ARIES. We did, however, wonder if the Knight clock performance could have been improved had more training samples been used. Whereas the Knight training dataset consisted of six cohorts with 207 samples, the testing dataset consisted of $>1000$ samples. We also wondered if the Knight clock consisting of nearly as many CpG sites (148) as training samples (207) might have suffered from overfitting.

To explore these concerns, we derived a new GA clock by fitting elastic nets implementing in the glmnet $\mathrm{R}$ package to the publicly available subset of the Knight testing and training data restricted to the same $27 \mathrm{~K} \mathrm{CpG}$ 
sites (total $n=400$; GSE36642 $n=51$; GSE62924 $n=38$; GSE79056 $n=36$; GSE80310 $n=24$; GSE66459 $n=22$; GSE69633 $n=46$; VICS $n=183$ ). The resulting clock included $193 \mathrm{CpG}$ sites, and its correlation with GA in ARIES was quite similar to the Knight estimate $(R=0.56)$. Both the original Knight clock and our new clock were correlated with measured GA $(R=0.37)$ and with the Bohlin estimate at similar strength $(R=0.49)$. Both GAA estimates were also similar $(R=0.49)$ and were correlated with the Bohlin GAA estimate at similar strength $(R=0.35)$. However, the new GAA estimate was more strongly correlated with birth weight ( $R=0.09 ; p=0.008)$ compared to the Knight estimate $(R=0.055, p=0.11)$.

Although our clock was less likely to suffer from overfitting (193 CpG sites from 400 samples), we still considered the ratio of $\mathrm{CpG}$ sites to training samples to be quite high. To investigate, we also generated clocks restricted to 50, 25, and $10 \mathrm{CpG}$ sites. The resulting clocks were still strongly associated with GA $(R=0.4,0.33$, and 0.25 , respectively) and with the default $193-\mathrm{CpG}$ clock $(R=0.82$, 0.72 , and 0.61 , respectively). GAA estimates were similarly strongly associated with the GAA estimate of the default clock $(R=0.79,0.68$, and 0.57 , respectively) and with birth weight $(R=0.12,0.12$, and 0.11 , respectively). These results show that $50 \mathrm{CpG}$ sites was sufficient to produce GA and GAA estimates with optimal performance.

To conclude, care must be taken when deriving and testing molecular models. Test dataset characteristics should match the datasets where the model will be later applied. Training datasets should be as large as possible. Although it is necessary to reserve data for testing in order to assess performance, it would be useful if authors also published a model trained using all available data. In most cases, the model derived from the large dataset will be superior. Finally, model sizes should be restricted to reflect the size of the training data to avoid overfitting.

\begin{abstract}
Funding
This work was supported by a grant from the UK Economic and Social Research Council [ES/N000382/1]. LDH is supported by a Career Development Award fellowship from the UK Medical Research Council [MR/ M020894/1]. AJS, MS, and LDH work in a unit that receives funding from the University of Bristol and the UK Medical Research Council [MC_UU_12013/2, MC_UU_12013/5, MC_UU_12013/9]. The UK Medical Research Council and the Wellcome Trust [Grant ref.: 102215/2/13/2] and the University of Bristol provide core support for ALSPAC.
\end{abstract}

\section{Availability of data and materials}

The datasets generated and/or analyzed during the current study are available from the corresponding author on reasonable request, subject to the ALSPAC study executive data access procedures, as specified on the ALSPAC website.

\section{Authors' contributions}

AJS and MS conducted statistical analyses; All authors contributed to writing and redrafting the manuscript and approved the final version.
Ethics approval and consent to participate

Ethics approval for the ALSPAC study was obtained from the ALSPAC Law and Ethics Committee and the Local Research Ethics Committees.

Participants provided written consent to participate.

\section{Consent for publication}

Not applicable.

\section{Competing interests}

The authors declare that they have no competing interests.

\section{Publisher's Note}

Springer Nature remains neutral with regard to jurisdictional claims in published maps and institutional affiliations.

Received: 1 September 2017 Accepted: 5 September 2017

Published online: 15 September 2017

References

1. Bohlin J, et al. Prediction of gestational age based on genome-wide differentially methylated regions. Genome Biol. 2016;17(1):207.

2. Knight AK, et al. An epigenetic clock for gestational age at birth based on blood methylation data. Genome Biol. 2016;17(1):206.

3. Horvath S. DNA methylation age of human tissues and cell types. Genome Biol. 2013;14(10):R115.

4. Chen $\mathrm{BH}$, et al. DNA methylation-based measures of biological age: metaanalysis predicting time to death. Aging (Albany NY). 2016;8(9):1844-65.

5. Girchenko $P$, et al. Associations between maternal risk factors of adverse pregnancy and birth outcomes and the offspring epigenetic clock of gestational age at birth. Clin Epigenetics. 2017:9:49.

6. Relton $\mathrm{CL}$, et al. Data resource profile: accessible resource for integrated epigenomic studies (ARIES). Int J Epidemiol. 2015;44(4):1181-90.

Submit your next manuscript to BioMed Central and we will help you at every step:

- We accept pre-submission inquiries

- Our selector tool helps you to find the most relevant journal

- We provide round the clock customer support

- Convenient online submission

- Thorough peer review

- Inclusion in PubMed and all major indexing services

- Maximum visibility for your research

Submit your manuscript at www.biomedcentral.com/submit 